\title{
Experimental validation of tangential resolution improvement in photoacoustic tomography using modified delay-and-sum reconstruction algorithm
}

Sandeep Kumar Kalva Manojit Pramanik 


\title{
Experimental validation of tangential resolution improvement in photoacoustic tomography using modified delay-and-sum reconstruction algorithm
}

\author{
Sandeep Kumar Kalva and Manojit Pramanik* \\ Nanyang Technological University, School of Chemical and Biomedical Engineering, 62 Nanyang Drive, Singapore 637459, Singapore
}

\begin{abstract}
For a circular scanning geometry in photoacoustic tomography, the axial/radial resolution is spatially invariant and is not affected by the ultrasound transducer (UST, detector) aperture. However, the tangential resolution is dependent on the detector aperture size and it varies spatially. Many techniques were proposed to improve the tangential resolution, such as attaching a concave lens in front of the nonfocused transducer or using a virtual point detector. Both of these methods have difficulties. Therefore, a modified delay-and-sum reconstruction algorithm has been proposed which can be used together with a standard ultrasound detector (nonfocused) to improve the tangential resolution. In this work, we validate the modified delay-and-sum algorithm experimentally for both flat and cylindrically focused USTs. More than threefold improvement in tangential resolution is observed. It is also shown that the object shape is recovered with this modified algorithm, which is very helpful for diagnosis and treatment purposes. @ 2016 Society of Photo-Optical Instrumentation Engineers (SPIE) [DOI: 10.1117/1.JBO .21.8.086011]
\end{abstract}

Keywords: image reconstruction; tangential resolution; photoacoustic tomography; thermoacoustic tomography.

Paper 160265R received Apr. 21, 2016; accepted for publication Aug. 3, 2016; published online Aug. 22, 2016.

\section{Introduction}

Photoacoustic imaging (PAI) is an emerging hybrid biomedical imaging modality combining light and sound. ${ }^{1}$ It combines the high spatial resolution of ultrasonic imaging and high contrast of optical imaging. PAI has various clinical applications such as molecular imaging, ${ }^{2}$ breast cancer imaging, ${ }^{3,4}$ brain imaging, ${ }^{5}$ sentinel lymph node imaging, ${ }^{6-8}$ vasculature imaging, ${ }^{9}$ temperature monitoring, ${ }^{10}$ tissue engineering, ${ }^{11}$ cancer screening, ${ }^{12}$ tumor angiogenesis imaging, ${ }^{13}$ and so on. ${ }^{14,15}$ In PAI, the target object (e.g., biological tissue) is illuminated with pulsed laser light. When the incident light energy is absorbed by the tissue chromophores, such as red blood cells, melanin, and water, there is a local temperature rise, which leads to thermal expansion of the tissue and generation of pressure waves that are emitted in the form of ultrasonic waves [also known as photoacoustic (PA) waves]. These PA waves are detected using an ultrasound transducer (UST) around the tissue boundary.

In photoacoustic tomography (PAT), the PA waves are typically acquired in a circular geometry around the object boundary. From these boundary PA data, various reconstruction algorithms can be used for obtaining the initial pressure rise distribution inside the tissue. ${ }^{16-24}$ In a circular scanning PAT, axial resolution is along the radial direction and is spatially invariant, whereas tangential resolution is along the tangential direction and is spatially variant. ${ }^{25,26}$ The axial resolution is mainly dependent on the transducer bandwidth, whereas the tangential resolution is dependent on both transducer bandwidth as well as transducer aperture size (active area). When the target object is far from the scanning center (i.e., when it is closer to the detector surface), the tangential resolution is poorer for a detector with larger aperture. One of the easiest ways to get rid of this problem is to move the UST very far from the scanning center so

*Address all correspondence to: Manojit Pramanik, E-mail: manojit@ ntu.edu.sg that the imaging region is far from the detector surface. However, this increases the scanning radius unnecessarily and practically may not be feasible due to the space constraints. Also the further detector is moved, the more its sensitivity decreases. To improve the tangential resolution without increasing the scanning radius, a negative acoustic lens concept was proposed, which increases the acceptance angle of the large detector and improves the tangential resolution. ${ }^{27}$ There are some practical challenges with this method. Attaching the inhouse made acoustic lens to the detector surface without the formation of any air bubbles is difficult. Also absorption of ultrasound signal and the impedance mismatch between the negative lens and the acoustic coupling medium (water/mineral oil) results in loss of signal. Another problem is that it is very difficult to make an in-house acoustic lens for cylindrically focused detectors, which are also used in circular scanning PAT for better elevation resolution (slice thickness). ${ }^{28}$ A custom made detector with curved piezo surface or a negative lens attached to the piezo surface inside the transducer can overcome these problems associated with an in-house acoustic lens, but typically such custom made detectors are very expensive. Another method to improve tangential resolution is to employ high numerical aperture (NA)-based virtual point detectors that provide a wide acceptance angle, high sensitivity, and negligible aperture effect. ${ }^{29,30}$ However, these types of transducers are also home-made, and are not readily available for purchase from the market. So to overcome these difficulties, a modified delay-and-sum reconstruction method was proposed to improve the tangential resolution without using any negative acoustic lens or any custom made detectors. ${ }^{31}$ Simulations showed promising results for this modified delay-and-sum reconstruction algorithm together with conventional ultrasound detectors with large apertures. In 
this work, the modified delay-and-sum reconstruction algorithm is experimentally validated for various shaped phantoms. Additionally, it is demonstrated that the modified reconstruction algorithm can also be used with a cylindrically focused ultrasound detector. Both numerical simulations and experimental data are shown to validate the effectiveness of the modified delay-and-sum reconstruction method.

Three different types of phantoms were used for demonstration purposes: (a) point sources (pencil leads of 0.5-mm diameter) placed at different distances from the scanning center, (b) large circular objects [low-density polyethylene (LDPE) tubes of 5-mm inner diameter filled with black Indian ink], and (c) "N"-shaped LDPE tubes (0.38-mm inner diameter) filled with mice blood to mimic blood vessels, embedded inside chicken tissue. Both flat and cylindrically focused transducers were used and showed that with modified delay-and-sum reconstruction method tangential resolution can be improved more than three times for both these transducers. Moreover, with modified reconstruction, the object shapes are also preserved.

\section{Materials and Methods}

\subsection{Modified Delay-and-Sum Reconstruction Algorithm}

The modified delay-and-sum reconstruction algorithm is given in detail elsewhere. ${ }^{31}$ The algorithm is briefly summarized here. For a delta light illumination $\delta(t)$, the initial pressure rise at a position $\vec{r}$ on a tissue is given by $p_{o}(\vec{r})=\Gamma(\vec{r}) A(\vec{r})$, where $A(\vec{r})$ is a spatial light absorption function and $\Gamma(\vec{r})$ is the Gruneisen parameter of the tissue. The main objective of the PAT image reconstruction is to estimate the initial pressure rise $p_{o}(\vec{r})$ inside the tissue from a set of measured acoustic signals $p\left(\vec{r}_{o}, t\right)$. The acoustic pressure $p\left(\vec{r}_{o}, t\right)$ at position $\vec{r}_{o}$ and time $t$, due to initial pressure source $p_{o}(\vec{r})$, obeys the following photoacoustic wave equation in an acoustically homogenous medium:

$\nabla^{2} p\left(\vec{r}_{o}, t\right)-\frac{1}{c^{2}} \frac{\partial^{2}}{\partial t^{2}} p\left(\vec{r}_{o}, t\right)=-p_{o}(\vec{r}) \frac{\partial \delta(t)}{\partial t}$,

where $c$ is the speed of sound. The initial pressure rise can be obtained by means of backprojection $\mathrm{as}^{20}$

$p_{o}(\vec{r})=\int b\left(\vec{r}_{o}, t=\frac{\left|\vec{r}-\vec{r}_{o}\right|}{c}\right) \frac{\mathrm{d} \Omega_{o}}{\Omega_{o}}$,

where $\Omega_{o}$ is the solid angle subtended by the entire surface $S_{o}$ with respect to $\vec{r}$ (reconstruction point inside $S_{o}$ ). $\Omega_{o}=2 \pi$ for a planar geometry and $\Omega_{o}=4 \pi$ for spherical and cylindrical geometries. $\mathrm{d} \Omega_{o}$ is the solid angle subtended by detection element $\mathrm{dS}_{o}$ with respect to reconstruction point at $\vec{r}$. The term $\mathrm{d} \Omega_{o} / \Omega_{o}$ is a weighting factor that contributes to the reconstruction from the detection element $\mathrm{dS}_{o}$ [Figs. 1 and 2 of Ref. 20]. $b\left(\vec{r}_{o}, t\right)$ is the backprojection term given by

$b\left(\vec{r}_{o}, t\right)=2 p\left(\vec{r}_{o}, t\right)-2 c t \frac{\partial p\left(\vec{r}_{o}, t\right)}{\partial t}$.

In the conventional delay-and-sum algorithm, the backprojection equation is implemented by recording the pressure wave as the backprojection term: $b\left(\vec{r}_{o}, t\right)=p\left(\vec{r}_{o}, t\right)$. For largeaperture detectors, the recorded pressure signal at $\vec{r}_{o}$ can be represented as a surface integral over the detector aperture ${ }^{26}$ $p^{\prime}\left(\vec{r}_{o}, t\right)=\iint p\left(\vec{r}_{o}^{\prime}, t\right) W\left(\vec{r}_{o}^{\prime}\right) \mathrm{d}^{2} \vec{r}_{o}^{\prime}$,

where $W\left(\vec{r}_{o}^{\prime}\right)$ is the weighting factor contribution from different elements of the detector surface. During conventional delayand-sum reconstruction, large-aperture detectors are considered as a point detector, typically at the center of the transducer surface. This introduces artifacts in the reconstructed image. In the modified delay-and-sum reconstruction algorithm, the entire surface area of the detector was considered during backprojection. The signal recorded in the transducer $\left[p^{\prime}\left(\vec{r}_{o}, t\right)\right]$ was backprojected from the entire surface of the detector instead of backprojecting from $\vec{r}_{o}$. So that the entire integrating surface used to receive the PA signal was used in the modified reconstruction method. ${ }^{31}$ Since all the simulations were done in two dimensions (2-D), the recording surface of the transducer is a line segment instead of a circular surface (Fig. 1 of Ref. 31). As such, many small segments on the line have been considered from which the recorded PA signal $p^{\prime}\left(\vec{r}_{o}, t\right)$ is backprojected instead of a single center point of the line. The same process is repeated for all transducer positions. For backprojecting, the first term of Eq. (3) was used for both conventional and modified delay-and-sum reconstruction techniques. All elements of the line contribute equally for detecting a signal (as the size of the small segments of the transducer is small). Therefore, the weighing factor $W\left(\vec{r}_{o}^{\prime}\right)$ was considered to be unity. For the experimental data, even though the recorded PA signals are in 3-D, all the reconstructions were done in 2D. Since the projection of the cylindrically focused transducer is also a line on a 2-D plane, the same algorithm can be used for the data acquired with cylindrically focused UST as well. For experimental validation, both flat USTs and cylindrically focused USTs have been used.

\subsection{Numerical Simulations}

Numerical simulations were done using $k$-wave tool box in MATLAB. ${ }^{32}$ The simulation geometry used is shown in Fig. 1(a). The computational grid was of $820 \times 820$ pixels $(0.1 \mathrm{~mm} /$ pixel $)$ with a perfectly matched boundary layer. Circular scanning geometry with $40 \mathrm{~mm}$ scanning radius was used. The imaging region was the circular shaded region (in blue) of radius $40 \mathrm{~mm}$ as shown in Fig. 1(a). PA data were acquired at 400 sensor locations by placing an UST on the scanning circle. We have used a $2.25-\mathrm{MHz}$ center frequency with $70 \%$ nominal bandwidth detectors. In all the simulations, sound speed was chosen as $1500 \mathrm{~m} / \mathrm{s}$. For simplicity, an ideal medium (acoustically homogeneous) was considered that exhibited no properties of absorption or dispersion of sound energy. Signal-to-noise ratio (SNR) was maintained at $40 \mathrm{~dB}$ by adding $1 \%$ noise to the simulated data. The three numerical phantoms used were point targets, circular shape, and $\mathrm{N}$-shaped blood vessel phantom [Figs. 1(b)-1(d)]. Figure 1(b) shows the five point targets used, located at 0,8 , 16,24 , and $32 \mathrm{~mm}$ from the scanning center. The second numerical phantom [Fig. 1(c)] consists of two circles of $5-\mathrm{mm}$ diameter located at 0 and $15 \mathrm{~mm}$ from the scanning center. The third numerical phantom [Fig. 1(d)] consists of an $\mathrm{N}$-shaped blood vessel numerical phantom of $0.38-\mathrm{mm}$ diameter. The simulated PA data were generated with a time step size of $40 \mathrm{~ns}$ and a total of 1250 time steps for point source numerical phantoms (1010 time steps for circular and blood vessel numerical phantoms). Once the forward PA data were 

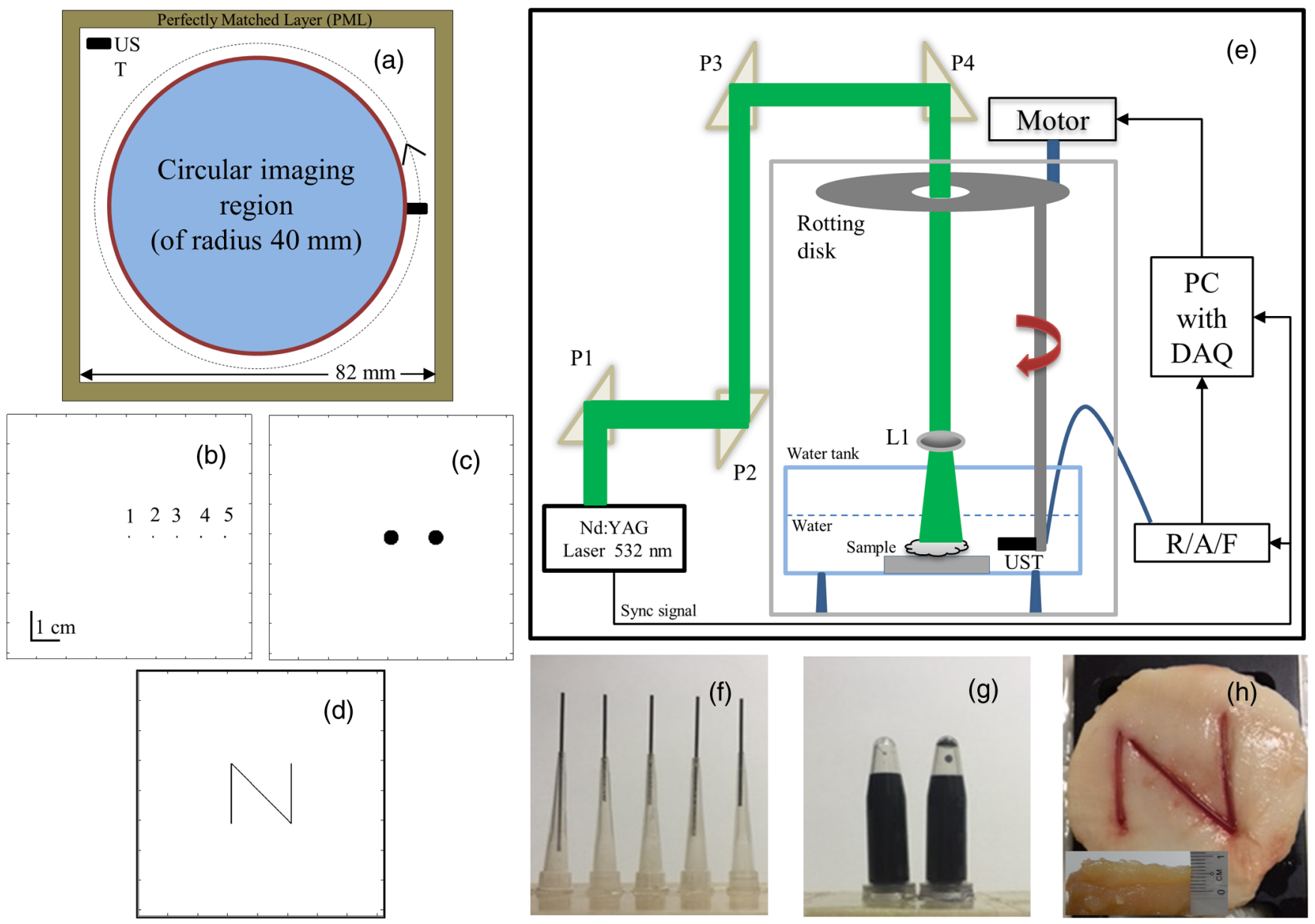

Fig. 1 (a) Schematic diagram of the $k$-wave simulation geometry in MATLAB. $820 \times 820$ pixels $(0.1 \mathrm{~mm} /$ pixel) was used for all the simulations. Scanning radius $40 \mathrm{~mm}$. UST: ultrasound transducer. (b) Point source numerical phantom. Five-point targets were placed at a distance of $0,8,16,24$, and $32 \mathrm{~mm}$ from the scanning center. (c) Circular-shaped numerical phantom (5-mm diameter): one at center and the other at $15 \mathrm{~mm}$ from the center. (d) N-shaped blood vessel numerical phantom (vessel width $0.38 \mathrm{~mm}$ ). (e) Schematic diagram of the experimental set up. DAQ: Data acquisition card, R/A/F: receiver, amplifier, and filter for photoacoustic signal. P1, P2, P3, and P4 are uncoated prisms. L1: Plano concave lens. Rotating disc: connected to UST and controlled by motor. The UST and the sample are immersed in water for ultrasound coupling. (f) Point source phantom made using pencil leads of $0.5-$ $\mathrm{mm}$ diameter. (g) Circular-shaped phantom made using LDPE tubes (5-mm diameter) filled with black Indian ink. (h) Blood vessel phantom of $\mathrm{N}$ shape made with LDPE tubes of 0.38-mm inner diameter filled with mice blood embedded inside chicken breast tissue. On top of this, another layer of chicken tissue of 6-mm thickness was placed as shown in inset.

generated using the $k$-wave, both traditional delay-and-sum and the modified delay-and-sum reconstruction methods were used for reconstructing the cross-sectional PAT images and further analysis were made. All reconstructions were done using a desktop with i7 Intel 64 bit processor $(3.4 \mathrm{GHz})$ and $8 \mathrm{~GB}$ RAM running the Windows 10 operating system.

\subsection{Experimental Method}

The schematic diagram of the experimental setup is shown in Fig. 1(e). A Q-switched Nd:YAG laser was used to deliver laser pulses of $10 \mathrm{~Hz}$ at 532-nm wavelength with a 5-ns laser pulse width. The laser energy density on the object was $\sim 3.18 \mathrm{~mJ} / \mathrm{cm}^{2}$, which is much lower than the ANSI safety limit $^{33}$ of $20 \mathrm{~mJ} / \mathrm{cm}^{2}$ at 532-nm wavelength. A nonfocused UST (Olympus NDT, V306-SU) and a cylindrically focused UST (Olympus NDT, V306-SU-NK, CF = 1.90 in.) with 13- $\mathrm{mm}$ diameter active area and 2.25-MHz central frequency with $\sim 70 \%$ nominal bandwidth were used to acquire the PA signal. The UST acquires the data around the sample, a full $360 \mathrm{deg}$ in circular configuration for a continuous data acquisition time of $480 \mathrm{~s}$ with a rotational speed of $0.75 \mathrm{deg} / \mathrm{s}$. The acquired PA signals were regrouped into 800 A-lines. ${ }^{34}$ The acquired PA signal was first amplified and filtered by a pulse amplifier (Olympus-NDT, 5072PR) and then recorded using a data acquisition card (GaGe, compuscope 4227) inside a desktop. All PA data were acquired with $25-\mathrm{MHz}$ sampling rate.

The phantoms used for the experiments were as shown in Figs. 1(f)-1(h). The first phantom was the point source phantom consisting of five pencil leads $(0.5 \mathrm{~mm})$ held using pipette adhered on an acrylic slab [Fig. 1(f)]. The leads were placed at a distance of $0,8,16,24$, and $32 \mathrm{~mm}$ from the scanning center. The second phantom was a circular-shaped phantom made using LDPE tubes of 5-mm inner diameter filled with black Indian ink 

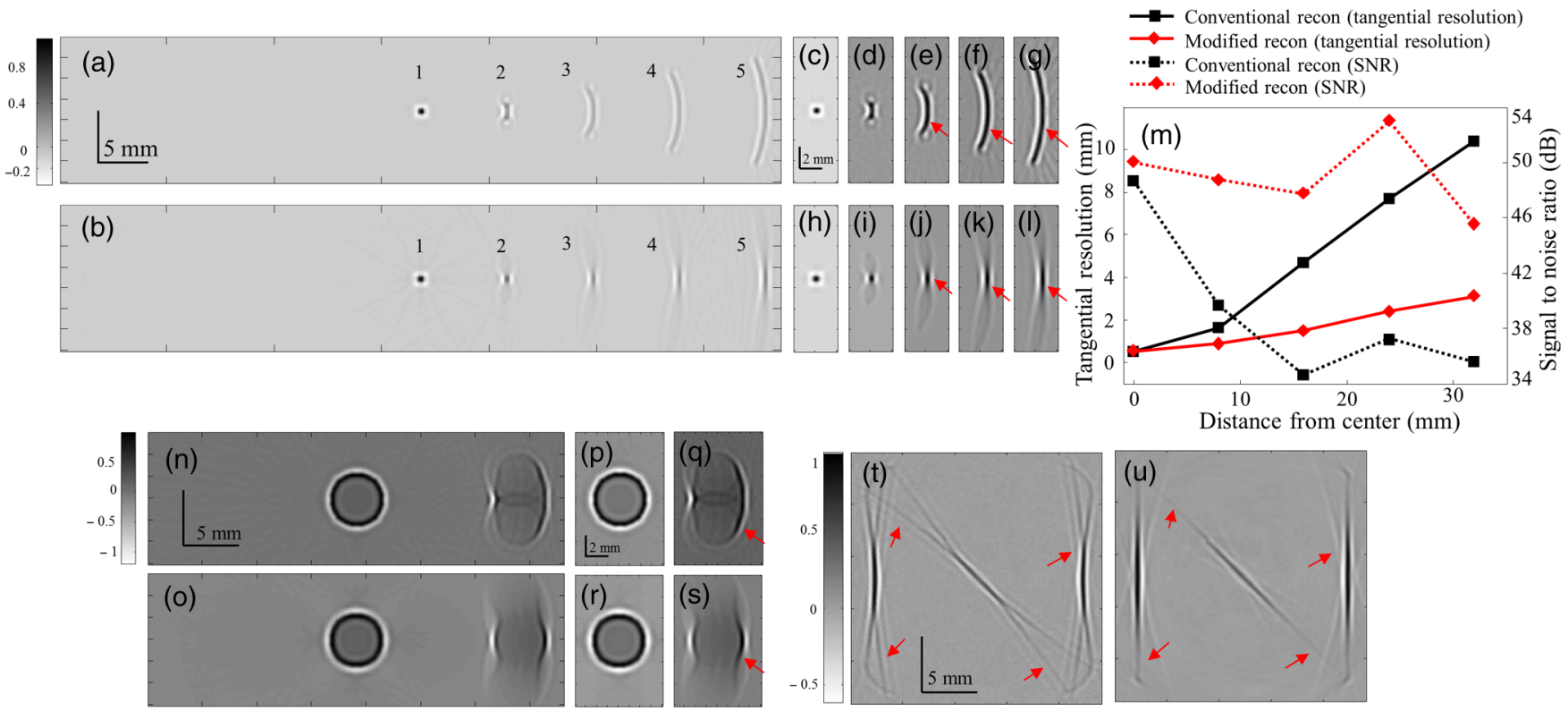

Fig. 2 (a)-(l) Simulation results for point source phantoms. (a) Conventionally reconstructed PAT images of 5-point targets. (b) Reconstructed using modified delay-and-sum reconstruction algorithm. (c)-(g) Zoomed in point targets 1 to 5 in (a). (h)-(l) Zoomed in point targets 1 to 5 in (b). (m) Comparison of the tangential resolution and SNR between conventional and modified reconstruction algorithm as a function of distances from the scanning center. (n)-(s) Simulation results for circularshaped numerical phantom. (n) Conventionally reconstructed PAT images of two circles at different distances from scanning center. (o) Reconstructed modified reconstruction algorithm. (p) and (q) Zoomed in individual circles in (n). ( $r$ ) and (s) Zoomed in individual circles in (o). (t) Conventionally reconstructed PAT image of $\mathrm{N}$-shaped blood vessel numerical phantom. (u) Reconstructed using modified reconstruction. Red arrow points to the places where improvements can be visible clearly.

[Fig. $1(\mathrm{~g})]$. The tubes were placed at 0 and $15 \mathrm{~mm}$ from the scanning center and attached to an acrylic slab at the bottom. The third phantom was a blood vessel phantom made using LDPE tubes of $0.38-\mathrm{mm}$ inner diameter filled with mice blood embedded inside a layer of chicken breast tissue in the form of an $\mathrm{N}$ shape [Fig. 1(h)]. On top of this layer, another chicken breast tissue layer of 6-mm thickness was placed as shown in Fig. 1(h) inset. All samples were placed inside the water bath for better ultrasound coupling. Once again, after the PA data acquisition, both traditional delay-and-sum and the modified delay-and-sum reconstruction methods were used for reconstructing the cross-sectional PAT images and further analysis was made. The tangential resolution improvement was shown quantitatively. The tangential resolution was calculated as the full width at half maximum (FWHM) of the point spread function along the tangential direction. The SNR was also calculated for all the targets from the reconstructed PAT images. The SNR is defined as the amplitude of the PA signal from the target divided by the standard deviation of the background noise SNR $=V / n$, where $V$ is the PA signal amplitude from the target, and $n$ is the standard deviation of the background noise.

\section{Results and Discussions}

\subsection{Simulation Results}

Figure 2 shows the reconstructed images of three numerical phantoms using conventional reconstruction and modified reconstruction algorithms. Figure 2(a) shows the conventionally reconstructed PAT images of the five point targets. Figures $2(\mathrm{c})-2(\mathrm{~g})$ show the zoomed in images of point targets 1 to 5 , respectively. As can be clearly seen from these reconstructed PAT crosssectional images, there is an elongation of the point target in the tangential direction. The further the point is from the scanning center (or the closer the point to the UST), the larger the elongation (or poorer the tangential resolution). Figure 2(b) shows the reconstructed PAT images using the modified delay-and-sum algorithm. It is evident that with modified reconstruction, the elongation along the tangential direction has been reduced. Figures 2(h)-2(l) show the zoomed in images of the point targets 1 to 5, respectively. From the zoomed in images, it is even clearer that with modified reconstruction, the tangential elongation is drastically improved. Figure $2(\mathrm{~m})$ shows the tangential resolution and SNR at each point target position for Figs. 2(a) and 2(b). From this comparison plot, more than three times improvement in terms of tangential resolution and a relatively higher SNR is observed depending on the location of the point target in the scanning region. So, in addition to the improvement in tangential resolution, SNR was also improved with modified reconstruction algorithm.

Next, it was demonstrated that the modified delay-and-sum reconstruction algorithm preserves the shape of the target object. For this, a numerical phantom with two large circles was used. Figure 2(n) shows the conventionally reconstructed PAT images of the two circles, one at the scanning center and the other at $1.5 \mathrm{~cm}$ from the scanning center. Figure 2(o) shows the PAT images reconstructed using modified delay-and-sum. As can be observed from the zoomed in images of each circle [Figs. 2(p)-2(s)], the shape of the circular target object has been distorted in the tangential direction (the further the object from the scanning center or the nearer the object to the UST, the 
greater is the degradation). After using the modified reconstruction algorithm, it was noticed that the shape of the object was preserved as shown in Figs. 2(q) and 2(s). The distortion in the tangential direction for the circular object has been reduced. SNR improvement can also be observed here. For the circle at the scanning center using the conventional reconstruction algorithm, SNR was calculated to be $41.36 \mathrm{~dB}$ and using the modified reconstruction algorithm it was $45.58 \mathrm{~dB}$. For the circle $1.5 \mathrm{~cm}$ away from the scanning center, SNR was calculated to be 34.68 and $35.32 \mathrm{~dB}$ using traditional and modified reconstruction techniques, respectively. Comparatively, the SNR was better apart from preserving the target shape with the modified reconstruction algorithm.

Finally, a numerical phantom in the shape of an N was simulated and reconstructed using the conventional and modified reconstruction algorithms as shown in Figs. 2(t) and 2(u). This phantom was used to mimic the blood vessel. It can be observed that the shape has been retained in the modified reconstruction technique (red arrows). In this numerical phantom, also, there was no SNR degradation (28.49 dB using traditional and $28.68 \mathrm{~dB}$ using modified reconstruction algorithms). Therefore, all the simulation results were demonstrated that the modified delay-and-sum reconstruction algorithm can help to improve the tangential resolution without using any external lens or other techniques proposed earlier, without any compromise in the SNR values. In Sec. 3.2, it was demonstrated that this algorithm can be applied in real-experimental data as well.

\subsection{Experimental Results}

As described earlier, three different types of phantoms were used for the experimental work as well. PAT data were collected for all the objects with two different USTs, both flat as well as cylindrically focused USTs. The PAT images of the sample were reconstructed using both conventional reconstruction algorithm and modified delay-and-sum reconstruction algorithm. Figure 3(a) shows the conventionally reconstructed PAT images of the point targets (pencil leads located at 0, 8, 16, 24, and $32 \mathrm{~mm}$ from the scanning center) obtained using an unfocused UST. As can be seen in the zoomed images [Figs. 3(c)-3(g)], the target object elongation increases in the tangential direction when it is nearer to the detector surface or away from the scanning center. But with the modified reconstruction algorithm, improvement was evident from the zoomed images [Figs. 3(h)3(1)]. There was a more than threefold improvement in the tangential resolution. The quantified tangential resolution and SNR values versus distances from the scanning center are shown in Fig. 3(m). It can be clearly seen that the modified reconstruction technique improves the tangential resolution without any
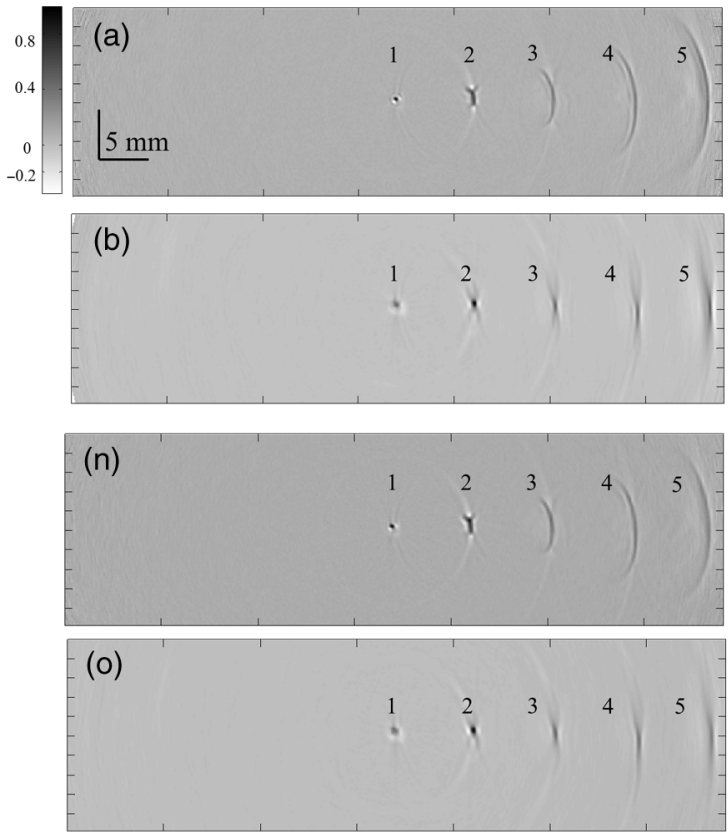
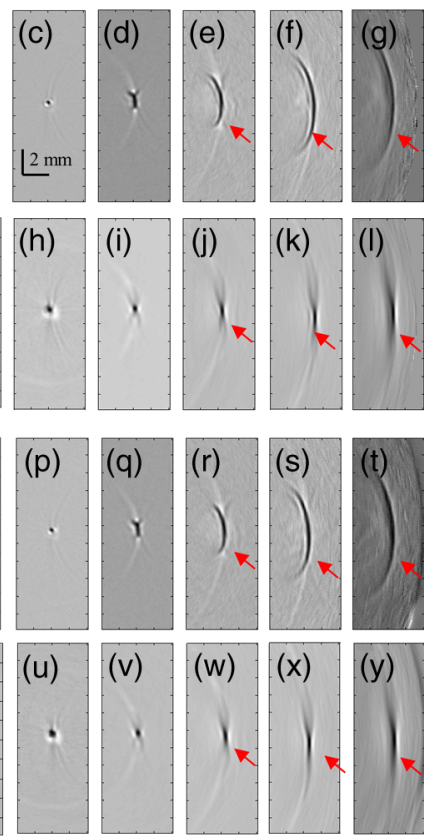
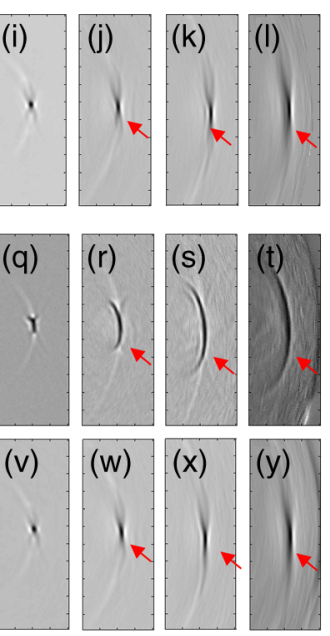
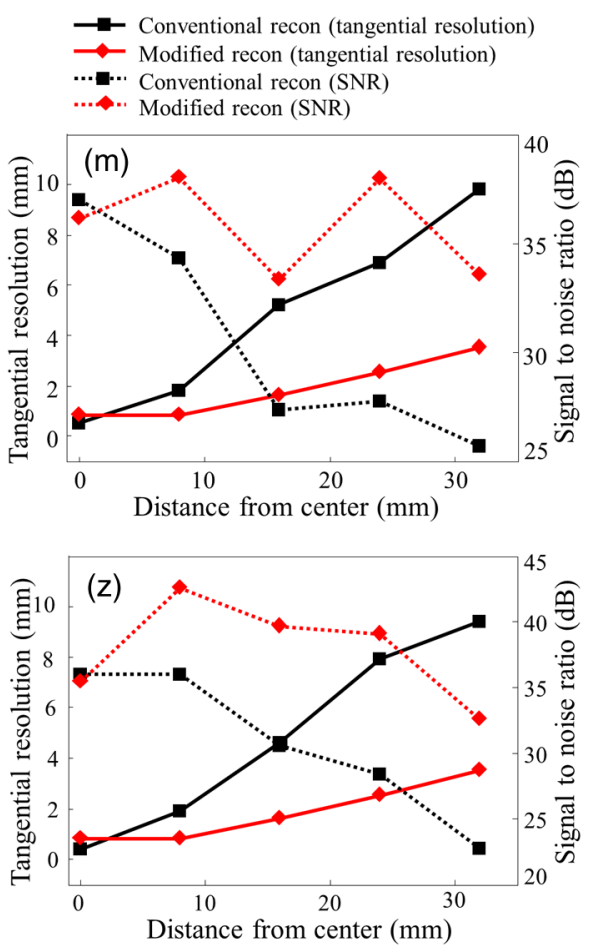

Fig. 3 (a)-(I) Experimental results using unfocused UST of $2.25 \mathrm{MHz}$ for point source phantoms. (a) Conventionally reconstructed PAT images of 5-point targets (b) Reconstructed using modified delay-and-sum reconstruction algorithm. (c)-(g) Zoomed in point targets 1 to 5 in (a). (h)-(l): Zoomed in point targets 1 to 5 in (b). (m) Comparison of tangential resolution and SNR between conventional and modified reconstruction algorithm as a function of distances from the scanning center for unfocused UST. (n)-(y) Experimental results using cylindrically focused UST of $2.25 \mathrm{MHz}$ for point source phantom. (n) Conventionally reconstructed PAT images. (o) Reconstructed using modified reconstruction algorithm. (p)-(t) Zoomed in point targets 1 to 5 in (n). (u)-(y) Zoomed in point targets 1 to 5 in (o). (z) Comparison of tangential resolution and SNR between conventional and modified reconstruction algorithm as a function of distances from the scanning center for cylindrically focused UST. Red arrows point to the places where improvements can be visible clearly. 
compromise in the SNR values compared to the conventional reconstruction technique. Experiments were repeated for the same pencil leads configuration using a cylindrically focused UST. A similar trend in tangential resolution can be observed as shown in Figs. 3(n)-3(z). For the cylindrically focused UST, we observed more than threefold improvement in terms of tangential resolution and higher SNR. It is clearly shown that using this modified reconstruction algorithm we obtained a better SNR in addition to the improvement in tangential resolution.

Next, it was shown that with this modified reconstruction algorithm, the shape of the target object can also be retained. LDPE tubes (5-mm inner diameter) were filled with black Indian ink, one placed near the scanning center and the other at $\sim 1.5 \mathrm{~cm}$ from the scanning center. Figures $4(\mathrm{a})$ and $4(\mathrm{~b})$ show the reconstructed PAT images using conventional and modified reconstruction algorithms for the data collected using unfocused UST. In Fig. 4(d), there is a shape distortion for the circular object which is reconstructed conventionally. From Fig. 4(f), the shape preservation can be observed when the modified reconstruction algorithm was used. Similarly, for the data collected using cylindrically focused UST, shape of the target object was preserved [Figs. 4(g)-4(1)]. In addition to preserving the shape of the target, we were able to maintain the SNR levels. In the case of unfocused UST, SNR was 33.42 and $34 \mathrm{~dB}$ using conventional and modified reconstruction algorithms, respectively, for the tube at the center. For the tube at $15 \mathrm{~mm}$ away from the center, the SNR was 37.41 and $32.76 \mathrm{~dB}$ using conventional and modified reconstruction techniques, respectively. Similarly, in the case of cylindrically focused UST, for the tube at the scanning center, SNR was 29.58 and $30.8 \mathrm{~dB}$ using traditional and modified delay-andsum reconstruction, respectively. For the tube at $1.5 \mathrm{~cm}$ away from the center, SNR was 41.54 and $35.17 \mathrm{~dB}$, respectively, using conventional and modified reconstruction algorithms.

Next, a study was conducted to demonstrate that this modified reconstruction algorithm can be used for more realistic phantom imaging with tissue samples. For this study, LDPE tubes of 0.38-mm inner diameter filled with mice blood were embedded inside a chicken breast tissue that was cut into a circular shape [Fig. 1(h)]. The tubes were embedded inside the tissue to form a blood vessel network in an $\mathrm{N}$ shape of $2 \mathrm{~cm} \times 2 \mathrm{~cm}$ dimensions. On top of this layer of tissue, a layer of 6-mm thick chicken breast was placed. Figures 5(a) and 5(b) show the PAT images reconstructed using conventional and modified reconstruction techniques, respectively, for the
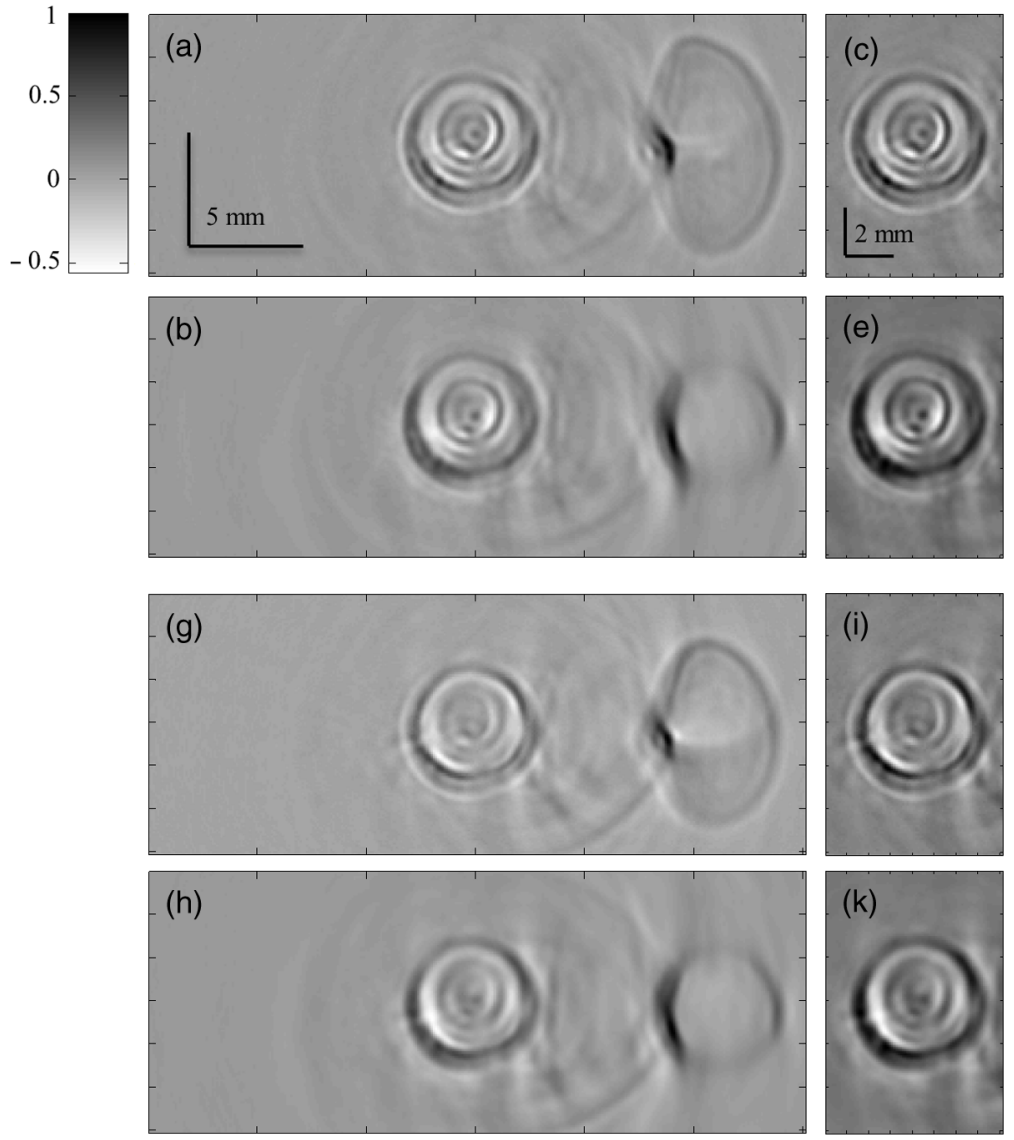
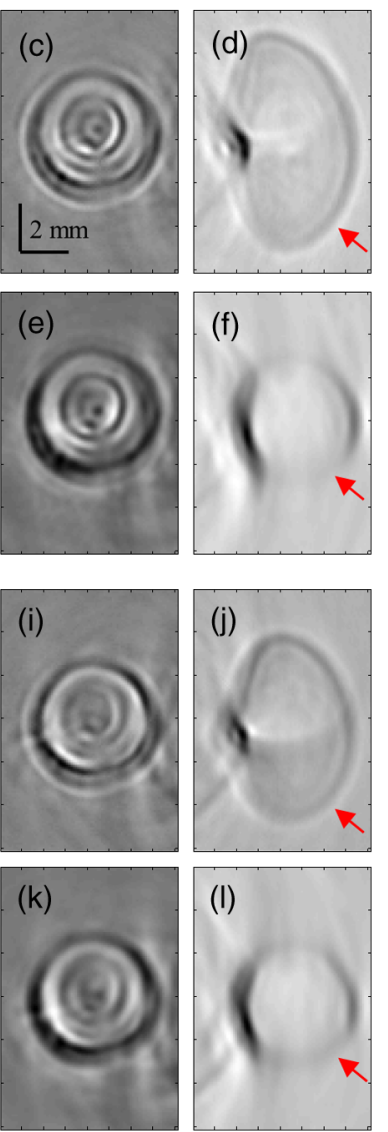

Fig. 4 (a)-(f) Experimental results using unfocused UST of $2.25 \mathrm{MHz}$ for large circular phantom (LDPE tube of 5-mm inner diameter filled with India black ink). (a) Conventionally reconstructed PAT images. (b) Using modified reconstruction algorithm. (c) and (d) Zoomed in individual circles in (a). (e) and (f): Zoomed in individual circles in (b). (g)-(l) Experimental results using cylindrically focused UST of $2.25 \mathrm{MHz}$ for the same phantom. (g) Conventionally reconstructed PAT images. (h) Reconstructed using modified reconstruction algorithm. (i) and (j) Zoomed in individual circles in (g). (k) and (l) Zoomed in individual circles in (h). Red arrows point to the places where improvements can be visible clearly. 

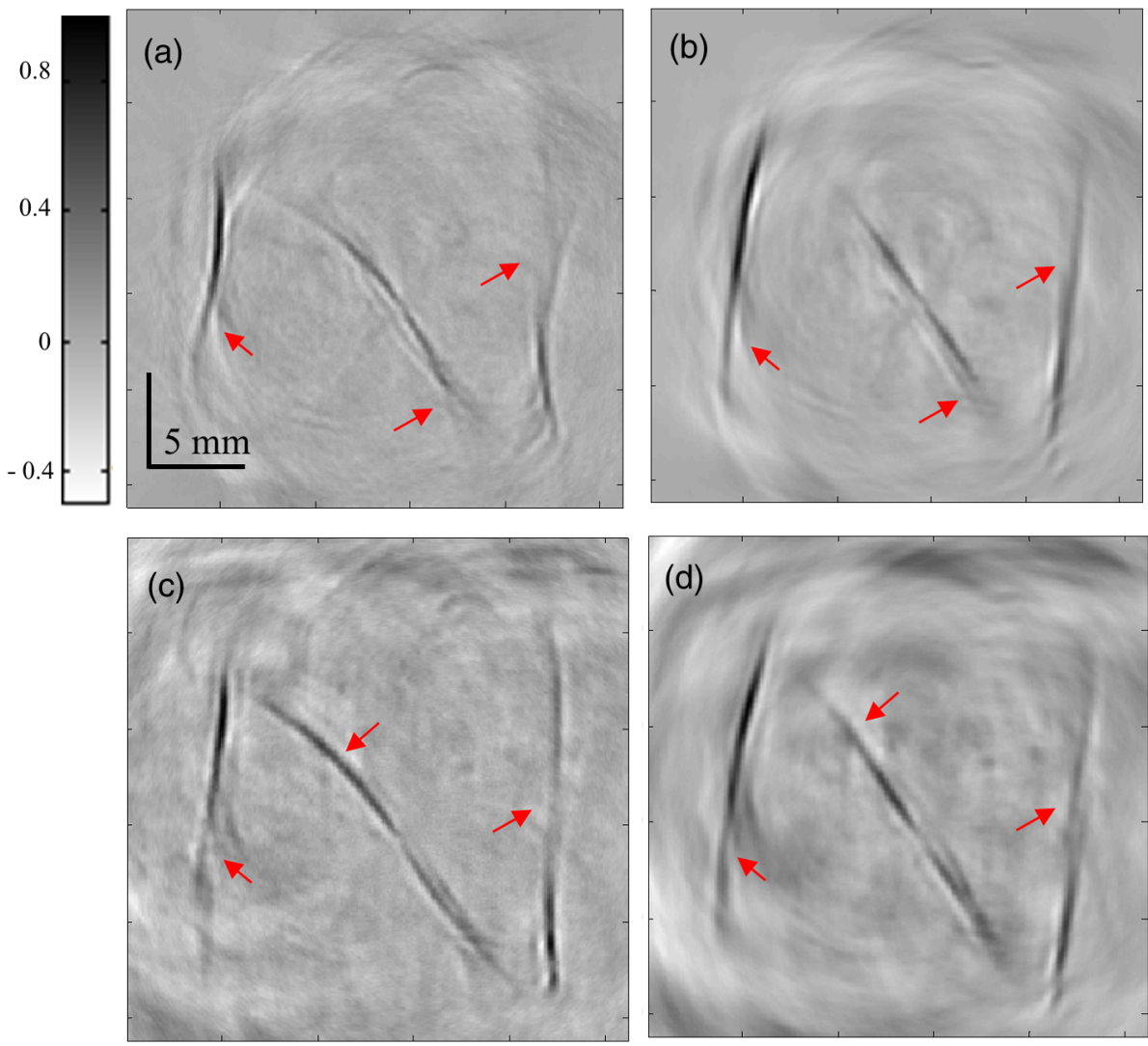

Fig. 5 (a) and (b) Experimental results for N-shaped blood vessel network (LDPE tube of 0.38-mm inner diameter filled with mice blood placed within chicken tissue) using unfocused UST. (a) Reconstructed PAT images with conventional reconstruction algorithm. (b) Using modified reconstruction algorithm. (c) and (d) Experimental results for the same phantom using cylindrically focused UST. (c) Using conventional reconstruction algorithm. (d) Using modified reconstruction algorithm. Red arrows point to the places where improvements can be visible clearly.

data collected using unfocused UST. Figures 5(c) and 5(d) show the corresponding PAT images for the cylindrically focused UST. From Figs. 5(a) and 5(c), we can notice that there is a distortion (curved and blurred lines) in the $\mathrm{N}$ shape as indicated by the red arrows. Improvement of shape can be seen in the reconstructed images in Figs. 5(b) and 5(d). There is no curving of the lines in the $\mathrm{N}$ shape and also we can see the entire shape of the tubes (corresponding red arrows). The distortion in the shape of the blood vessel phantom was reduced in the modified reconstructed PAT images and the SNR was also maintained. From the reconstructed cross-sectional PA images of the blood vessel network in tissue phantom, SNR was calculated to be $22.96,22.54 \mathrm{~dB}$ in the case of unfocused UST and $21.62,21.79 \mathrm{~dB}$ in the case of cylindrically focused UST, using standard and modified delay-and-sum reconstruction algorithms, respectively.

Hence, this modified delay-and-sum algorithm helps in improving the tangential resolution of the object nearer to the detector surface (further from the scanning center). There is no requirement for attaching any negative acoustic lens to the detector surface or use of any virtual point detectors. Moreover, there is no loss of signal due to the presence of the lens. For the 800 A-lines data, the reconstruction time using the modified delay-and-sum reconstruction algorithm was around $\sim 43$ min using the desktop configuration mentioned earlier. Reconstruction time can be reduced to $\sim 21 \mathrm{~min}$ if 400-A lines data are used. The reconstruction time can further be reduced if a larger grid size is used during reconstruction. For example, with 400-A line data and $0.2 \mathrm{~mm} /$ pixel grid size (instead of $0.1 \mathrm{~mm} /$ pixel used in all our work), the reconstruction time is only $\sim 2.5 \mathrm{~min}$. In case of the traditional reconstruction method, the reconstructions are very fast $[\sim 23 \mathrm{~s}$ for 800 A-lines data, and $\sim 12 \mathrm{~s}$ for 400 A lines data for $0.1 \mathrm{~mm} /$ pixel grid size]. Due to the nature of the modified algorithm, where at each UST location, the data are backprojected from many tiny section of the detector surface, the reconstruction time is longer than the standard delay-and-sum algorithm. However, this could be improved even further and made real time if reconstruction is done in $\mathrm{C} / \mathrm{C}++$ instead of MATLAB, as inherently MATLAB is not optimum for running loops. Another way to improve the reconstruction time is the use of graphics processor units (GPU). In the future, our goal will be to minimize this reconstruction time.

\section{Conclusion}

By using a modified delay-and-sum reconstruction algorithm, the tangential resolution of PAT can be improved more than threefold for both flat as well as cylindrically focused USTs. The reconstruction algorithm can also be used for thermoacoustic tomography (TAT), where instead of a laser irradiation source, a pulsed microwave source is generally used. We have shown both simulation results and experimental validation that the modified reconstruction is effective. Three types of 
phantoms (both numerical and experimental) were used, namely point target, large circular object, and an $\mathrm{N}$-shaped blood vessel phantom. In all three different types of phantom, we observed improvements in terms of tangential resolution and SNR was maintained. Therefore, without using any external negative acoustic lens on the surface of the transducer, we can improve the tangential resolution without compromising the high SNR values.

\section{Acknowledgments}

This work was funded by the Ministry of Education in Singapore Tier 2 Research Grant (ARC2/15: M4020238). The authors would like to acknowledge Dr. Paul Kumar Upputuri for helping in the experimental setup and Dr. Li Liang, Dr. Jhonson, and Shok Li for providing mice blood to conduct experiments.

\section{References}

1. L. V. Wang and S. Hu, "Photoacoustic tomography: in vivo imaging from organelles to organs," Science 335(6075), 1458-1462 (2012).

2. D. Pan et al., "Molecular photoacoustic imaging of angiogenesis with integrin-targeted gold nanobeacons," FASEB J. 25(3), 875-882 (2011).

3. W. Xia et al., "Design and evaluation of a laboratory prototype system for 3D photoacoustic full breast tomography," Biomed. Opt. Express 4(11), 2555-2569 (2013).

4. M. Pramanik et al., "Design and evaluation of a novel breast cancer detection system combining both thermoacoustic (TA) and photoacoustic (PA) tomography," Med. Phys. 35(6), 2218 (2008).

5. X. Wang et al., "Three-dimensional laser-induced photoacoustic tomography of mouse brain with the skin and skull intact," Opt. Lett. 28(19), 1739-1741 (2003).

6. D. Pan et al., "Near infrared photoacoustic detection of sentinel lymph nodes with gold nanobeacons," Biomaterials 31(14), 4088-4093 (2010).

7. T. N. Erpelding et al., "Sentinel lymph nodes in the rat: noninvasive photoacoustic and US imaging with a clinical US system 1," Radiology 256(1), 102-110 (2010).

8. M. Pramanik et al., "In vivo carbon nanotube-enhanced non-invasive photoacoustic mapping of the sentinel lymph node," Phys. Med. Biol. 54(11), 3291-3301 (2009).

9. S. Hu and L. V. Wang, "Photoacoustic imaging and characterization of the microvasculature," J. Biomed. Opt. 15(1), 011101 (2010).

10. M. Pramanik and L. V. Wang, "Thermoacoustic and photoacoustic sensing of temperature," J. Biomed. Opt. 14(5), 054024 (2009).

11. Y. Talukdar et al., "Multimodal ultrasound-photoacoustic imaging of tissue engineering scaffolds and blood oxygen saturation in and around the scaffolds," Tissue Eng. Part C Methods 20(5), 440-449 (2014).

12. T. Wang et al., "Characterization of ovarian tissue based on quantitative analysis of photoacoustic microscopy images," Biomed. Opt. Express 4(12), 2763-2768 (2013).

13. X. Wang et al., "Noninvasive laser-induced photoacoustic tomography for structural and functional in vivo imaging of the brain," Nat. Biotechnol. 21(7), 803-806 (2003).

14. K. Daoudi et al., "Handheld probe integrating laser diode and ultrasound transducer array for ultrasound/photoacoustic dual modality imaging," Opt. Express 22(21), 26365 (2014).

15. X. Cai et al., "Photoacoustic tomography of foreign bodies in soft biological tissue," J. Biomed. Opt. 16(4), 046017 (2011).

16. C. Lutzweiler, X. L. Dean-Ben, and D. Razansky, "Expediting modelbased optoacoustic reconstructions with tomographic symmetries," Med. Phys. 41(1), 013302 (2014).

17. J. Prakash et al., "Basis pursuit deconvolution for improving modelbased reconstructed images in photoacoustic tomography," Biomed. Opt. Express 5(5), 1363 (2014).
18. C. Huang et al., "Full-wave iterative image reconstruction in photoacoustic tomography with acoustically inhomogeneous media," IEEE Trans. Med. Imaging 32(6), 1097-1110 (2013).

19. C. B. Shaw et al., "Least squares QR-based decomposition provides an efficient way of computing optimal regularization parameter in photoacoustic tomography," J. Biomed. Opt. 18(8), 080501 (2013).

20. M. Xu and L. V. Wang, "Universal back-projection algorithm for photoacoustic computed tomography," Phys. Rev. E 71(1), 016706 (2005).

21. K. P. Köstli and P. C. Beard, "Two-dimensional photoacoustic imaging by use of Fourier-transform image reconstruction and a detector with an anisotropic response," Appl. Opt. 42(10), 1899-1908 (2003).

22. M. Xu and L. V. Wang, "Pulsed-microwave-induced thermoacoustic tomography: filtered backprojection in a circular measurement configuration," Med. Phys. 29(8), 1661-1669 (2002).

23. M. Xu and L. V. Wang, "Time-domain reconstruction for thermoacoustic tomography in a spherical geometry," IEEE Trans. Med. Imaging 21(7), 814-822 (2002).

24. G. Paltauf et al., "Iterative reconstruction algorithm for optoacoustic imaging," J. Acoust. Soc. Am. 112(4), 1536 (2002).

25. M. Haltmeier and G. Zangerl, "Spatial resolution in photoacoustic tomography: effects of detector size and detector bandwidth," Inverse Prob. 26(12), 125002 (2010).

26. M. Xu and L. V. Wang, "Analytic explanation of spatial resolution related to bandwidth and detector aperture size in thermoacoustic or photoacoustic reconstruction,” Phys. Rev. E 67(5), 056605 (2003).

27. M. Pramanik, G. Ku, and L. V. Wang, "Tangential resolution improvement in thermoacoustic and photoacoustic tomography using a negative acoustic lens," J. Biomed. Opt. 14(2), 024028 (2009).

28. D. Queirós et al., "Modeling the shape of cylindrically focused transducers in three-dimensional optoacoustic tomography," J. Biomed. Opt. 18(7), 076014 (2013)

29. C. Li and L. V. Wang, "Photoacoustic tomography of the mouse cerebral cortex with a high-numerical-aperture-based virtual point detector," J. Biomed. Opt. 14(2), 024047 (2009).

30. C. Li and L. V. Wang, "High-numerical-aperture-based virtual point detectors for photoacoustic tomography," Appl. Phys. Lett. 93(3), 33902 (2008).

31. M. Pramanik, "Improving tangential resolution with a modified delayand-sum reconstruction algorithm in photoacoustic and thermoacoustic tomography," J. Opt. Soc. Am. A 31(3), 621-627 (2014).

32. B. E. Treeby and B. T. Cox, "k-Wave: MATLAB toolbox for the simulation and reconstruction of photoacoustic wave fields," J. Biomed. Opt. 15(2), 021314 (2010).

33. American National Standard for Safe Use of Lasers, ANSI Z136.12000.

34. P. K. Upputuri and M. Pramanik, "Performance characterization of lowcost, high-speed, portable pulsed laser diode photoacoustic tomography (PLD-PAT) system,” Biomed. Opt. Express 6(10), 4118-4129 (2015).

Sandeep Kumar Kalva received his master's degree in biomedical engineering from the Indian Institute of Technology (IIT), Hyderabad, India, in 2015 . He is currently a PhD student in the School of Chemical and Biomedical Engineering, Nanyang Technological University, Singapore. His research area is on functional photoacoustic imaging for various clinical applications.

Manojit Pramanik received his $\mathrm{PhD}$ in biomedical engineering from Washington University in St. Louis, Missouri, USA. He is an assistant professor of the School of Chemical and Biomedical Engineering, Nanyang Technological University, Singapore. His research interests include the development of photoacoustic/thermoacoustic imaging systems, image reconstruction methods, clinical application areas such as breast cancer imaging, molecular imaging, contrast agent development, light transport through biological tissue, Monte Carlo simulation of light propagation in biological tissue, and Monte Carlo simulation of Raman scattering. 